\title{
PHOTOMETRIC UNITS AND NOMENCLATURE
}

\author{
By Edward B. Rosa
}

\section{CONTENTS}

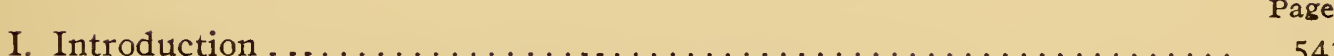

II. General Discussion and Derivation of Formulas . . . . . . . . . . . . . . 544

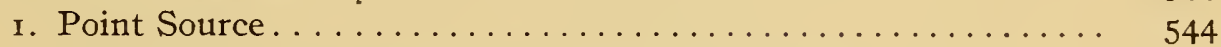

2. Distinction between Luminous Flux and Energy . . . . . . . . 546

3. Definition of Intensity for Unsymmetrical Sources ......... 547

4. Unit Disk .............................. 548

5. Extended Source-Circular Disk................. 550

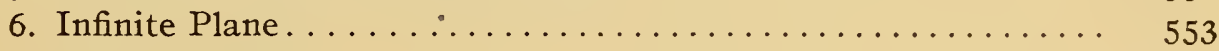

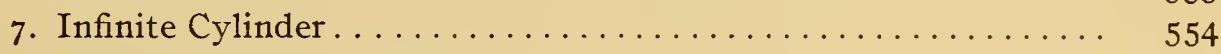

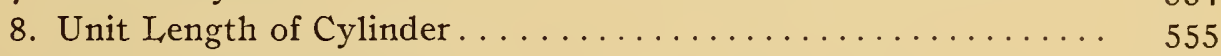

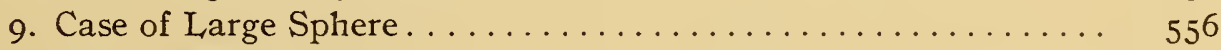

Io. Reciprocal Relations....................... 558

I I. Hollow Sphere . . . . . . . . . . . . . . . . . . 560

12. Luminous Flux within an Inclosure ............... 56 I

III. Summary of Photometric Relations . . . . . . . . . . . . . . 562

IV. Problems for Illustrations . . . . . . . . . . . . . . . . . . . 569

V. Collection of Formulas ........................... 57 I

\section{INTRODUCTION}

The subject of photometric units and nomenclature received a notable impulse by the paper of Blondel, ${ }^{1}$ presented to the Geneva Congress of 1896 . Since that time various modifications in the proposals there made have been put forward, and the units and nomenclature there proposed have come into use to a greater or less degree. There has, however, been a tendency to recognize as few separate photometric quantities as possible, and some of them have been employed rather loosely in more than one sense. This

${ }^{1}$ A. Blondel, Rapport sur les Unités Photometriques, L'Eclairage Electrique, Vol. 6, pp. $148-157 ; 1896$. 
is partly, at least, due to a lack of clearness in the perception of the physical relation of the various photometric quantities.

The Illuminating Engineering Society appointed a committee ${ }^{2}$ on this subject something over a year ago, and it was due to my membership in this committee that I was led to give the matter careful attention.

The following paper is an attempt at a systematic discussion of the mathematical and physical relationships of the various photometric quantities. Some of the units that have been objected to are found to be most useful and to contribute materially to clear thinking and concise expression. Although many of the theorems derived below are not new, they are nevertheless useful in developing the desired relations between the various photometric quantities. Acknowledgment is made to Blondel, Palaz, Liebenthal, Hering, Kennelly, Sharp, Hyde, Jones, and others, whose writings and discussions have done much to develop the subject.

In what follows, some of the names are used in a different sense from what has been usual, and slight changes have been made in some of the symbols. These are in the interest of a more systematic arrangement and also of international uniformity.

\section{GENERAL DISCUSSION AND DERIVATION OF FORIMULAS}

\section{POINT SOURCE}

We start with the idea of light as a luminous flux, radiating or flowing away from the source and illuminating bodies as it falls upon them. In the simple case of a point source the flux is equal in all directions, and since the entire flux falls uniformly upon the interior surface of any concentric sphere the quantity of the luminous flux per unit of area is inversely proportional to the square of the distance, a law which has been verified by experiment. The quantity of the luminous flux per unit of area or the flux density at the surface of the illuminated body is by definition the illumination $E$. If we represent the total flux by $F$, we have therefore

$$
E=\frac{F}{4 \pi r^{2}}
$$

\footnotetext{
2 The committee consists of Dr. C. H. Sharp, chairman, Prof. A. E. Kennelly, Prof. E. L. Nichols, Prof. A. Blondel, and the writer.
} 
where $r$ is the distance from the point source to the body illuminated.

Representing $\frac{F}{4 \pi}$ by a single letter $I$, we have

$$
E=\frac{I}{r^{2}}
$$

and

$$
F=4 \pi I
$$

$I$ is called the intensity of the source, and is equal to the flux per unit of solid angle.

The illumination is equal to the intensity of the source divided by the square of the distance (2), and the total flux is $4 \pi$ times the intensity (3).

The intensity $I$ is measured in candles, ${ }^{3}$ the flux $F$ in lumens, and the distance $r$ in centimeters. In practice $r$ is often measured in meters or in feet. Thus from a point source of intensity $I$ candles there is a luminous flux of $4 \pi I$ lumens.

The flux density is the luminous flux per unit of area normal to the flux in the case of a point source, or the total flux $F$ over an area divided by the area $S$; thus the flux density is $\frac{F}{S}$, or $\frac{d F}{d S}$ when it is variable.

If the source is not a point but a small sphere of radius $a$, the flux $4 \pi I$ passes out from a radiant surface $4 \pi a^{2}$. Thus, the flux density of radiation or the specific radiation, is

$$
\frac{F_{s}}{S}=\frac{4 \pi I}{4 \pi a^{2}}=\frac{I}{a^{2}}=E^{\prime}
$$

Thus, we may speak generally of the luminous radiation at any point in space, and of the flux density of such radiation. If it falls on a material surface the incident flux density is the illumination $E$; as it comes from a luminous or other radiating or diffusing surface, the flux density is the radiation, $E^{\prime}$. Although $E$ and $E^{\prime}$ are quantities of the same nature, it is convenient thus to distinguish them.

${ }^{3}$ It is proposed to call the new value of the American candle, which is the same as the English candle and the French bougie decimale, and which is also used by several other countries, the international candle. 
The luminous flux density in space is analogous to electric displacement in electrostatics. We think of an electric displacement as occurring in space between two or more electric charges, but a surface density occurs only where there is a material conducting body on which the lines of electric force terminate. In the same way the terms luminous flux and flux density apply generally both at the surface of the luminous and the illuminated bodies and in the space between. The radiation is the flux density at the source of the flux, and the illumination is the flux density or flux per unit of area on the surface where the luminous flux is received.

\section{DISTINCTION BETWEEN LUMINOUS FLUX AND ENERGY}

The total luminous flux $F$ is not to be confused with the total energy flowing from a luminous body. Luminous flux, or light as we ordinarily say, is the physical stimulus which applied to the retina produces the sensation of light. It is equal to the radiant power multiplied by the stimulus coefficient. This stimulus coefficient is different for every different wave frequency or wave length, and is of course zero for all frequencies outside the visible spectrum. Hence, if $W_{\lambda}$ is the power (expressed in watts) for unit of wave length of the spectrum, and $K_{\lambda}$ is the stimulus coefficient or luminous efficiency whose value varies with the wave length $\lambda$, we have for the total power radiated from a body

$$
W=\int_{0}^{\infty} W_{\lambda} d \lambda
$$

the integration being carried through the whole range of wave lengths, including of course the non-luminous radiation.

For the luminous flux

$$
F=\int K_{\lambda} W_{\lambda} d \lambda
$$

the integration being throughout the visible spectrum, $K$ being zero elsewhere.

As the values of $K_{\lambda}$ throughout the spectrum are not accurately known, it is not possible to calculate $F$ in general. But by measuring $W$ in watts and $F$ in lumens, we can determine the ratio of the luminous flux to the radiant power, in any particular 
case. One may properly say that luminous flux is due to and is always associated with radiant power; but luminous flux and radiant power can not in general be converted into one another like feet and inches; for, as stated above, the conversion factor, the stimulus coefficient, or luminous efficiency, is not a constant like the ratio of feet to inches, but is variable, having a different value for every different wave length, in the visible spectrum and falling to zero outside the visible spectrum. "Luminous energy" should not therefore be used as synonymous with luminous flux.

\section{DEFINITION OF INTENSITY FOR UNSYMMETRICAL SOURCES}

For a point source the intensity $I$ has been defined as the total flux $F$ divided by $4 \pi$. If the source is not symmetrical, but sends out a total luminous flux $F$ unequally in different directions, then the mean value of the intensity is called the mean spherical intensity, and its value is

$$
I_{s}=\frac{F}{4 \pi}
$$

We thus define the mean spherical intensity with respect to the total flux; and similarly, the intensity $I$ in any particular direction is the ratio of the flux through a small solid angle in that direction to the angle. Thus

$$
\begin{aligned}
I & =\frac{F}{\omega}, \omega \text { being a solid angle, } \\
\text { or, } I & =\frac{d F}{d \omega}, d \omega \text { being an infinitesimal solid angle. }
\end{aligned}
$$

Thus the intensity $I$ is the angular density of the flux as the illumination $E$ is surface density. Therefore both $E$ and $I$ are flux ratios, lumens per unit area and lumens per unit solid angle respectively. One lumen per square meter is the lux, and one lumen per unit solid angle is a candle.

In the case of a point source or unit sphere radiating equally in all directions, the intensity $I$ is defined as the flux through a unit of solid angle, or steradian. That is, $I=F$ when $\omega=\mathrm{I}$. This is an angle subtended by $\frac{I}{4 \pi}$ of a spherical surface, and in the case 
where the solid angle is a circular cone, its section through the apex is a plane angle of $65^{\circ} 32^{\prime} 28^{\prime \prime}$.

\section{UNIT DISK}

Concerning a body charged with electricity, we have the two ideas, (I) the electricity of density $\sigma$ and total quantity $Q=\int \sigma d S$ on the surface of the charged body, and (2) the flux of force throughout the surrounding space, there being $4 \pi Q$ lines of force for a quantity $Q$ of electricity. We do not believe in the fluid theory of electricity in the same way that Franklin did, but we nevertheless find the idea of a surface density of electricity very useful. In the corresponding case with light we may have similarly two distinct ideas, (I) a surface distribution of light over a luminous area of brightness or specific quantity $b$, and total quantity $Q=\int b d S$, and (2) a luminous flux filling the surrounding space and producing an illumination $E$ on any body equal to the flux per unit of area, or $E=\frac{F}{S}$.

We have so far defined illumination and intensity in terms of the flux. Let us now obtain their values in terms of the quantity of light on the surface of the luminous source.

The illumination from a very small source is inversely proportional to the square of the distance from the source and directly proportional to the brightness of the source.

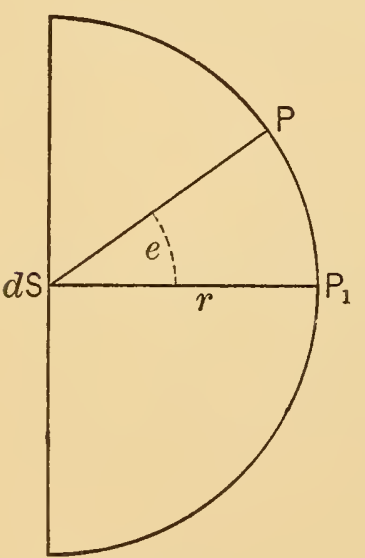

Fig. 1. Hence for a luminous plane of area $d S$ (Fig. I) we may write

$$
E_{\mathrm{P}_{1}}=\frac{Q}{r^{2}}=\frac{b d S}{r^{2}}
$$

where $Q$ is the total quantity of light on the disk, and the radiation to $P_{1}$ at a distance $r$ is normal. For a point $P$ at an angle $e$ from the normal the illumination would be

$$
E_{\mathrm{P}}=\frac{b d S \cos e}{r^{2}}=\frac{Q \cos e}{r^{2}}
$$


The total flux over the hemisphere illuminated by the disk is

$$
\begin{aligned}
& F=\int_{0}^{\frac{\pi}{2}} E_{\mathrm{P} 2 \pi r^{2}} \sin e d e=Q \int \frac{2 \pi r^{2} \sin e \cos e d e}{r^{2}} d e \\
& F=\left[\pi Q \sin ^{2} e\right]_{0}^{\frac{\pi}{2}}=\pi Q
\end{aligned}
$$

Thus the total luminous flux $F$ from a small plane disk is $\pi$ times the quantity of light $Q$ on the disk. ${ }^{4}$

The average illumination over the hemisphere of radius $r$ is $\frac{F}{2 \pi r^{2}}=\frac{1}{2} \frac{Q}{r^{2}}$, whereas the maximum illumination $E_{n}$ normal to the disk is $\frac{Q}{r^{2}}$. Thus the mean is half the maximum. The intensity $I$ has been defined as the angular rate of flux in any particular direction. It is, therefore, proportional to the illumination produced in the given direction. Thus in the case of the luminous disk we have

$$
\begin{aligned}
& I_{n}=\text { maximum intensity, (normal) }=Q \\
& I_{h}=\text { mean hemispherical intensity }=\frac{Q}{2} \\
& I_{s}=\text { mean spherical intensity }=\frac{Q}{4} \\
& \text { Thus } F=\pi I_{n}=4 \pi I_{s}
\end{aligned}
$$

That is, the intensity is numerically equal to the total quantity of light on the small disk for all points on the normal. It decreases to zero as we pass $90^{\circ}$ away from the normal, having a mean value of half the maximum for the whole hemisphere, and is on the average only one-fourth the maximum for the whole sphere. We may, therefore, say that the hemispherical reduction factor for the disk is one-half, and the mean spherical reduction factor is one-fourth, the disk being supposed luminous on one side only.

\footnotetext{
${ }^{4}$ In electrostatics the total flux is $4 \pi$ times the quantity $Q$. The difference is due, first, to the fact that the luminous disk is supposed luminous on one side only and hence there is radiation only on one side, whereas the electric flux would be on both sides; secondly, the cosine law makes the average flux only half what it would be if the factor $\cos e$ were omitted.
} 
Since the total flux $F$ from an area is $\pi Q$, where $Q$ is the quantity of light on the area, the flux from a unit of area is $\pi b$. This is the radiation $E^{\prime}$. Hence, in general

$$
E^{\prime}=\pi b
$$

For a small sphere of radius $a$, the total flux is

$$
\begin{aligned}
F & =E^{\prime} \times \text { surface } \\
& =\pi b \times 4 \pi a^{2}=\pi Q \\
\text { Also, } F & =4 \pi I \\
\therefore I & =\frac{Q}{4}
\end{aligned}
$$

That is, for a unit sphere ${ }^{5}$ the intensity is one-fourth the quantity of light on the sphere. If the distribution of light over the sphere is not uniform, the mean spherical intensity is still one-fourth the total quantity of light on the sphere, as it is also for a disk. In other words, a sphere produces the same illumination at a given point as a disk of the same diameter and same brightness placed so that the radiation from the disk to the point is normal.

\section{EXTENDED SOURCE-CIRCULAR DISK}

Let $d S$ be an element of a plane radiating surface of brightness $b$, defined by the equation

$$
Q=b S
$$

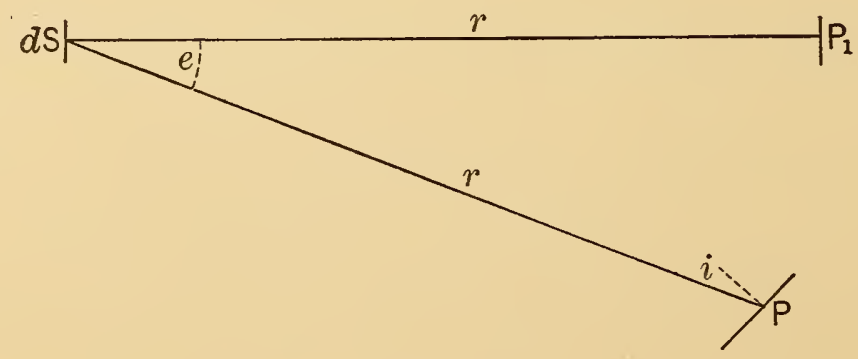

Fig. 2.

That is, the quantity of light $Q$ is equal to the product of $b$ into the surface $S ; b$, is the value of the quantity $Q$ when the surface is unity, and is the quantity of light per unit of area measured in

\footnotetext{
${ }^{5}$ By unit sphere or unit disk we mean a disk or sphere whose linear dimensions are negligible in comparison with the distance from source to receiver.
} 
candles. Thus, the intensity $I$ of such a source (or of any source) would be measured by comparing it experimentally with a standard light source, and it is equal to the intensity of a point source or unit sphere which produces the same illumination on a given test screen (of a photometer). Thus, while we define the intensity of a light source as the luminous flux per unit solid angle, we determine it by comparison with a concrete standard by means of the illumination produced on a test screen at a convenient distance, using a photometer and employing the law of inverse squares.

In Fig. 2 the illumination at $P_{1}$ in the normal to $d S$ is

$$
E_{1}=\frac{b d S}{r^{2}}
$$

while the illumination at $P$, the angles of emergence and incidence being $e$ and $i$ respectively is

$$
E_{2}=\frac{b d S \cos e \cos i}{r^{2}}
$$

The cosine law is assumed to hold exactly for both surfaces.

To calculate the illumination due to a large circular disk of brightness $b$ and any radius $a$ on a small plane area $P_{1}$, normal to the axis of the disk and situated on the axis at distance $r$ from the disk (see Fig. 3) we integrate the effect of each elementary circular ring of the disk. Thus, in equation (I4), putting $d S=$ $2 \pi x d x$,

$$
E=b \int_{0}^{a} \frac{2 \pi x d x \cdot \cos e \cos i}{\left(r^{2}+x^{2}\right)}
$$

Since $\cos e=\cos i=\frac{r}{\sqrt{r^{2}+x^{2}}}$

$$
\begin{aligned}
& \begin{aligned}
E & =\pi b \int_{0}^{a} \frac{2 x d x \cdot r^{2}}{\left(r^{2}+x^{2}\right)^{2}} \\
& =\pi b\left[\frac{-r^{2}}{r^{2}+x^{2}}\right]_{0}^{a}=\pi b\left[\mathrm{I}-\frac{r^{2}}{r^{2}+a^{2}}\right]
\end{aligned} \\
& \text { or, } E=\frac{\pi b a^{2}}{r^{2}+a^{2}}=\frac{b S}{r^{2}+a^{2}}=\frac{Q}{r^{2}+a^{2}}
\end{aligned}
$$

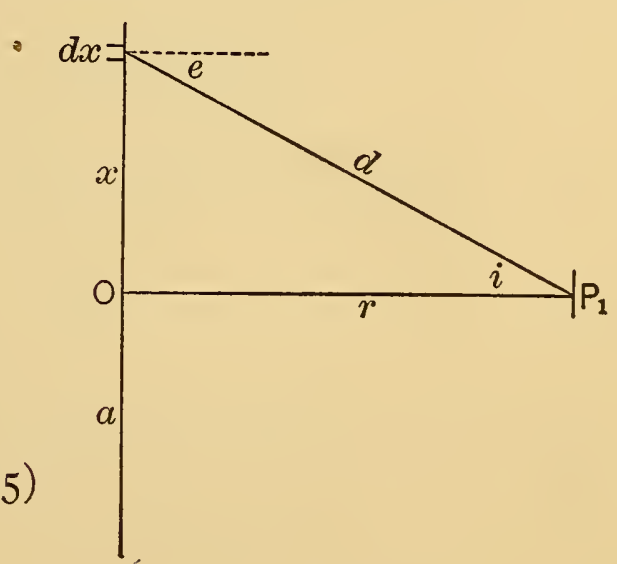

Fig. 3. 
where $Q$ is the product of the surface of the disk into the brightness $b$, and is the total quantity of light upon the disk measured in candles. If the disk were very small $Q$ would be the same as the maximum intensity $I_{n}$ of the source; but for an extended source we must distinguish between the equivalent intensity $I_{o}$ and the surface integral of the brightness $b$, which is $Q$. The latter we have called the quantity of light upon the disk; it is proportional to the total luminous flux $F$ coming from the extended source, and is equal to $F / \pi$, equation, (9). $Q$ and $F$ really measure the same thing, except that $Q$ is located on the source and is measured in candles, while $F$ is located in the surrounding space and is measured in lumens; their ratio is constant as $F=\pi Q$ always. ${ }^{6}$

In the case of the disk above mentioned, the illumination $E$ on a small plane normal to the axis is proportional to the total quantity of light $Q$ on the extended source (the circular disk) and inversely proportional to the square of the distance $d$ from $P_{1}$ to the edge of disk. This holds true for all distances $r$ from zero to infinity. Thus the law of inverse squares holds generally for the illumination along its axis due to a circular disk of any size, but the distance is measured, not to the center of the disk, but to the edge.

Thus we have

$$
E=\frac{I}{r^{2}} \text { for a point source or a unit disk, }
$$

${ }^{6}$ The total quantity of electricity on a disk of area $S$ is equal to the integral of the surface density $\sigma$ over the area. Thus

$$
\begin{aligned}
Q & =\int \sigma d S \\
& =\sigma S \text { when } \sigma \text { is uniform. }
\end{aligned}
$$

The brightness $b$ of a source corresponds to the surface density of electricity $\sigma$, and the total quantity of light over a surface is, in the same way, the surface integral of $b$. Thus

$$
\begin{aligned}
Q & =j b d S \\
& =b S \text { when } b \text { is uniform over the area } S .
\end{aligned}
$$

In the case of a sphere, the stirface $S=4 \pi a^{2}$. Therefore, for a spherical source $Q=4 \pi a^{2} b$, whereas the intensity $I=\pi a^{2} b$. That is, the intensity $I$ of a spherical source is one-fourth of $Q$, and is equal to the light on a disk of radius $a$ and brightness $b$. That is, the intensity of the sphere is equivalent to that of a disk of the same diameter and the same brightness for points at a great distance. 
To illustrate the rate of variation of the illumination with the distance, let $a=\mathrm{I}, r_{1}=\mathrm{I}, r_{2}=5$ (Fig. 4).

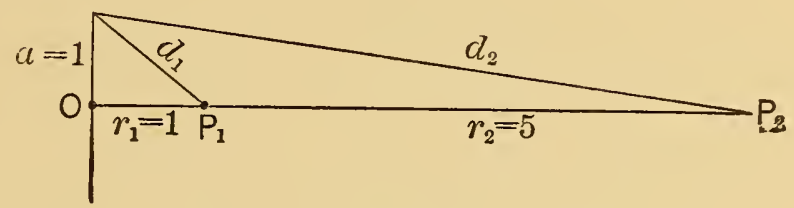

Fig. 4.

In the first case for the point $P_{1}, E_{1}=\frac{Q}{d_{1}^{2}}=\frac{\pi b}{2}$

In the second case for the point $P_{2}, E_{2}=\frac{Q}{d_{2}{ }^{2}}=\frac{\pi b}{26}$

Thus in the first case the distance is 5 times less and the illumination is 13 times more instead of 25 times more, as it would be if the light $Q$ were all concentrated at the center of the disk. If $r=0$, the illumination is $\pi b$ or twice as much as at $P_{1}$, and not infinite as it would be at zero distance from a point source.

This theorem is useful in measuring the radiation from walls, as the radiating area may be quite large and the photometer relatively near.

\section{INFINITE PLANE}

The radiation from an infinite plane $S$ (Fig. 5) upon a unit area of a pàrallel plane $T$ is found by integrating equation (I 5 ) to infinity. Thus

$$
E=\pi b \int_{0}^{\infty} \frac{2 x d x \cdot r^{2}}{\left(r^{2}+x^{2}\right)^{2}}=\pi b\left[\frac{-r^{2}}{r^{2}+x^{2}}\right]_{0}^{\infty}=\pi b
$$

Thus the flux density or illumination at any point $P$ on the $T$ plane is $\pi$ times the brightness $b$ on the radiating plane $S$ and is independent of the distance $r$.

From each unit of area of $S$ having a brightness $b$ the total flux is $\pi b$, as shown above. The

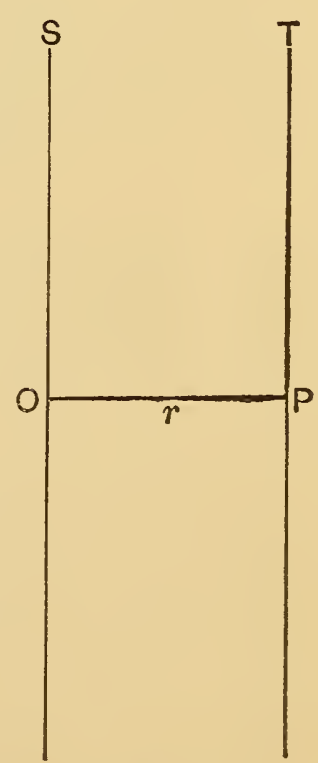

Fig. 5. resultant flux at all points is the same as though the total flux $\pi b$ from each unit of area of $S$ was confined to a cylindrical 
tube of unit area perpendicular to $S$, in which case the flux density would, of course, be constant at all sections-that is, at all distances (see Fig. 6).

\section{INFINITE CYLINDER}

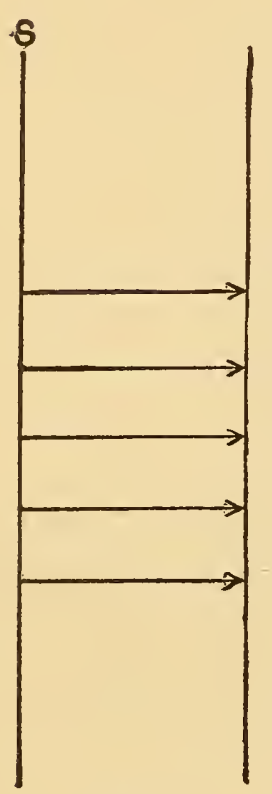

In a similar manner we may consider the flux from an infinite circular cylinder of uniform brightness $b$ and radius $a$.

The flux coming from unit length of the cylinder is $\pi b$ times the area. Hence $F=2 \pi^{2} a b$, whereas the flux falling on the inner surface of a concentric cylinder of radius $r$ is $E$ times the area, $E$ being the illumination. Hence for a unit of length of the cylinder $F=2 \pi r E$. Therefore,

$$
E=\frac{\pi a b}{r}=\mathrm{I} / 2 \frac{Q}{r}
$$

Thus the illumination due to an infinite cylinder varies inversely as the distance. This is interme-

Fig. 6. diate between the case of the point source, for which $E$ varies inversely as $r^{2}$, and the infinite plane, where $E$ is independent of the distance-that is, proportional to $r^{\circ}$.

The quantity $Q$ for the luminous cylinder is $b$ times the surface. Therefore the quantity per unit of length is

$$
Q_{1}=2 \pi a b
$$

The total luminous flux $F$, as stated above, is $2 \pi^{2} a b$. Hence the total flux per unit of length $F_{1}$ is $\pi$ times the quantity, or

$$
F_{1}=\pi Q_{1}
$$

or, for any portion (or the whole) of an infinite cylinder of uniform brightness, the total flux is $\pi$ times the quantity; that is,

$$
F=\pi Q
$$

as shown above for a circular disk. 


\section{UNIT LENGTH OF CYLINDER}

Suppose a light source in the form of a very long cylinder of radius $a$ and uniform brightness $b$. It is desired to determine experimentally its total luminous flux $F$. Suppose one has measured by means of a photometer the equivalent intensity $I_{1}$ of unit length of the cylinder (screening the photometer from all but a short section of the cylinder). We are to calculate the total flux $F_{1}$ from $I_{1}$. The unit length of cylinder will produce the same illumination at a distance as a rectangular plane of breadth $2 a$ and height unity of brightness $b$ equal to that of the surface of the cylinder. Hence the equivalent intensity $I_{1}$ is equal to $2 a b$ and the illumination produced on a photometer screen at distance $r$ is

$$
E=\frac{2 a b}{r^{2}}=\frac{I_{1}}{r^{2}}
$$

The quantity of light on the cylinder per unit of length is $b$ times the surface, or $2 \pi a b$, and the total flux $F_{1}$ is $\pi$ times the quantity. Thus we have

$$
\begin{aligned}
F_{1} & =2 \pi^{2} a b \\
I_{1} & =2 a b \\
\therefore F_{1} & =\pi^{2} I_{1}
\end{aligned}
$$

Thus, to obtain the total luminous flux $F_{1}$ from the measured value of the equivalent intensity of a unit of length of the luminous cylinder we multiply this intensity $I_{1}$ by $\pi^{2}$ instead of multiplying by $4 \pi$, as in the case of a sphere.

The spherical reduction factor of a short cylinder (the convex surface only being luminous) is therefore $\pi^{2} / 4 \pi=\pi / 4=0.785$. This would be nearly true for an incandescent lamp having one or more straight filaments. The value for a hairpin filament would be only slightly larger. Tantalum and tungsten lamps have reduction factors of about this value.

If the cylinder is long, we should then get the total flux $F$ by multiplying $F_{1}$ by the length of the cylinder. This demonstration is of course based on the assumption that the cosine law holds for the cylinder. If the source is a long tube, like the Moore light, the result would be subject to any modification dependent on its departure from the cosine law. 
Thus while the total fux $F$ is always $\pi$ times the quantity $Q$ of the source, it is not always $4 \pi$ times the intensity. It is $4 \pi$ times the intensity $I$ for a point source or sphere, $\pi^{2} L$ times the equivalent intensity $I_{1}$ (measured at a relatively great distance) of unit length of a long cylinder, $L$ being the length, and $\pi S$ times the equivalent intensity $I_{1}$ of unit of area of a plane, $S$ being the area of the plane.

It is, however, always $4 \pi$ times the mean spherical intensity of the given source. The illumination produced by a short cylinder is approximately inversely proportional to the square of the distance. For all distances greater than five times the length, the departures are not greater than 0.2 per cent in a particular case worked out by Hyde; the diameter of the cylinder in this case was one-tenth the length. The exact expression for the illumination due to a finite cylinder is not simple, and the calculation is tedious.

\section{CASE OF LARGE SPHERE}

If a surface $S_{1}$ (supposed a portion of a spherical surface of radius $r_{1}$ ) has a brightness $b$ and subtends a small solid angle $\omega$,

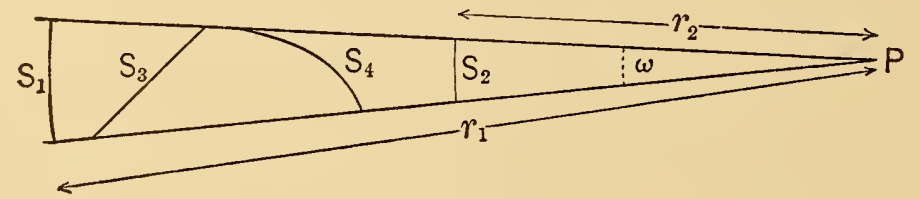

Fig. 7.

the illumination which it produces at $P$ (Fig. 7) is

$$
E=\frac{b S_{1}}{r_{1}^{2}}=\frac{b \omega r_{1}^{2}}{r_{1}^{2}}=b \omega
$$

A second surface $S_{2}$ of the same brightness will produce the same illumination at $P$ provided it subtends the same angle $\omega$. A third surface, $S_{3}$, at any angle will also produce the same illumination at $P$ if it has the same brightness $b$ and subtends the same solid angle $\omega$. For the radiation of each element $d S_{3}$ is $\frac{b d S_{3}}{r_{3}{ }^{2}} \cos e=\frac{b \omega r_{3}{ }^{2}}{r_{3}{ }^{2}}=b \omega$ as before. So also with the curved surface $\mathrm{S}_{4}$. In every case the greater distance from $P$ or the inclination of the angular position is compensated by the greater area included within the given (small) solid angle. 
Let us calculate the illumination at $P$ due to a large luminous sphere of radius $a$ and brightness $b, r$ being the distance from $P$ (Fig. 8) to the center of the sphere. Let the solid angle $A P B$ subtended at $P$ by the sphere be subdivided into a large number of elementary solid angles. Each of the latter incloses an area, as $S_{1}$, on the surface of the sphere, and also a corresponding area $S_{1}{ }^{\prime}$, on the circular disk $A B$. As we have just seen, the illumina-

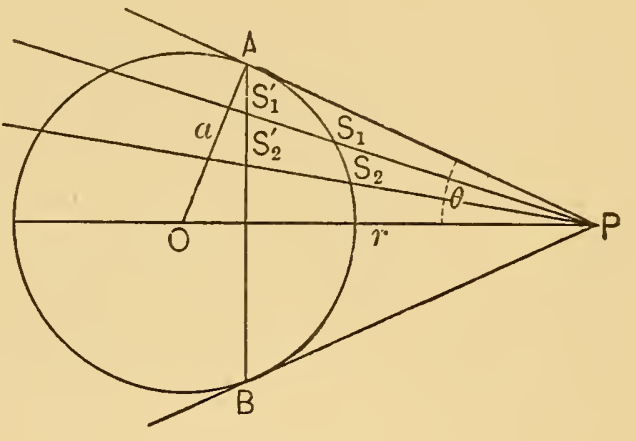

Fig. 8. tion produced at $P$ by each spherical area $S_{1}, S_{2}$, etc., is exactly the same as that produced by the corresponding plane areas $S_{1}{ }^{\prime}$, $S_{2}{ }^{\prime}$, etc., of the disk, if the brightness $b$ is the same for the disk as for the sphere. Therefore the illumination at $P$ due to the entire sphere is the same as that due to the disk $A B$, and we can calculate the latter by formula (I 5). That is,

$$
E=\frac{Q}{d^{2}}
$$

where $Q$ is the quantity of light on the disk and $d$ is the distance $A P$ from the point $P$ to the edge of the disk. $Q$ is equal to $b$ times the area of the disk, or

$$
\begin{aligned}
Q & =\pi(a \cos \theta)^{2} b \\
d & =r \cos \theta \\
\therefore E & =\frac{Q}{d^{2}}=\frac{\pi a^{2} b}{r^{2}} \\
& =\frac{I}{4} \frac{Q_{s}}{r^{2}}=\frac{I_{s}}{r^{2}}
\end{aligned}
$$

where $Q_{s}$ is the quantity of light on the sphere $=4 \pi a^{2} b$ and is constant for all distances, and $I_{s}$ is the intensity of the equivalent point source. Therefore the illumination produced by a sphere of any size is inversely proportional to the square of the distance measured from its center, and is equal to the intensity of a point source (or unit sphere) having the same total amount of light 
divided by the square of the distance. In other words, the inverse square law holds just as rigorously for large spheres as for

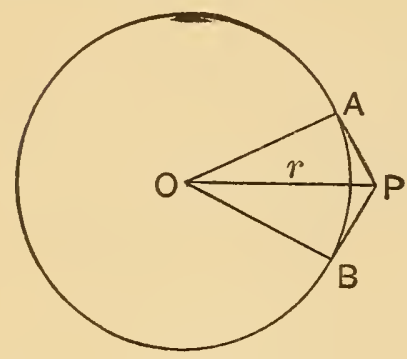

Fig. 9. points (always of course assuming the cosine law to hold for the spherical surfaces, and the brightness $b$ to be uniform over the sphere). When $P$ comes very near to the surface the area $A B$ of the sphere (Fig. 9) available for illuminating $P$ is very small, but the distance is just enough less to counterbalance. When $P$ comes up to the surface, $r=a$, and

$$
E=\pi b
$$

the same as for an infinite plane, to which the sphere is equivalent when the distance from the surface is reduced to zero.

The same result is reached more simply as follows:

A luminous sphere of radius $a$ and uniform brightness $b$ gives off a total flux $F=4 \pi a^{2} \times \pi b=4 \pi^{2} a^{2} b$. This produces an illumination on the inner surface of any concentric sphere, which by symmetry will be everywhere the same, and $F=4 \pi r^{2} E$.

$$
\therefore E=\frac{\pi a^{2} b}{r^{2}}=\frac{I}{r^{2}}
$$

Therefore the illumination produced by a sphere of uniform brightness is inversely proportional to the square of the distance from the center for all distances from the surface of the sphere to infinity.

\section{RECIPROCAL RELATIONS}

From what precedes we see that the illumination at any point $P$ due to the hollow hemisphere $A C B$ (Fig. Io) is the same as that due to the circular disk $A O B$. The latter is

$$
E=\frac{\pi a^{2} b}{A P^{2}}
$$

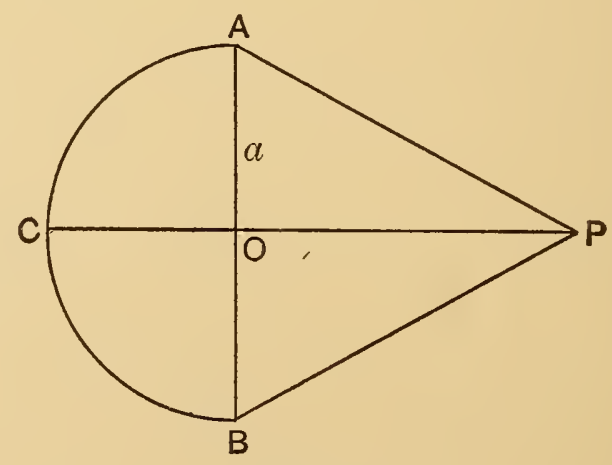

Fig. 10.

When $O P$ is reduced to zero the illumination due to the disk is $\pi b$, and hence the illumination at $O$ on an elementary plane area in 
the diametral plane is $\pi$ times the brightness $b$ of the surface of the sphere. We have already seen that the total flux from a unit of surface of brightness $b$ is $\pi b$. Hence the total flux through unit area $S$ at $O$, due to the hemisphere, is equal to the total flux through the hemisphere due to the luminous unit area $S$, the brightness $b$ being the same in each case.

This is a particular case of a more general proposition, namely, the flux due to any surface $S$ passing through an element dS is equal to the flux due to the latter passing through the former, the brightness being the same in each case.

As shown above, the illumination $E$ at $P_{1}$, due to $S_{1}$ (Fig. II) is equal to $b \omega$, where $b$ is the brightness of $S_{1}$ and $\omega$ is the (small) solid angle subtended at $P_{1}$ by $S_{1}$; this is independent of the shape of $S_{1}$ or its distance

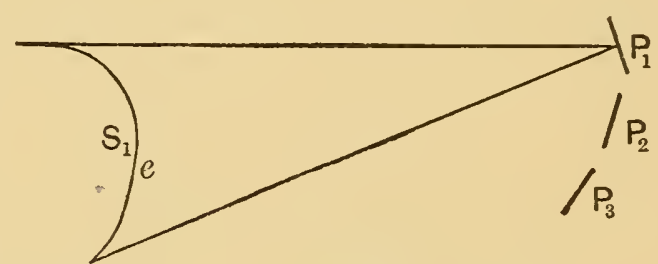

Fig 11. from $P_{1}$. The flux $F$ passing through $d S$ at $P_{1}$ is therefore

$$
F=\int b d \omega d S \cos \theta \text {, over the area of } S_{1}
$$

Or,

$$
F=b d S \int d \omega \cos \theta
$$
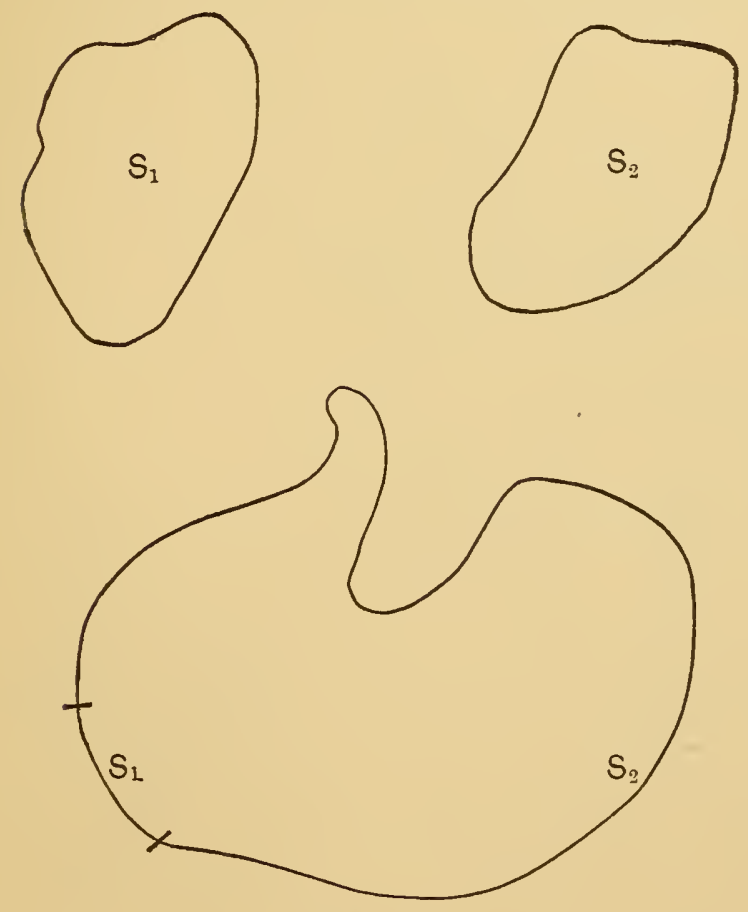

Fig. 12.

Similarly, the flux due to $d S$ at $P_{1}$ passing through $S_{1}$ is

$$
\begin{aligned}
F & =\int b d S \cos \theta d \omega \\
& =b d S \int \cos \theta d \omega
\end{aligned}
$$

In the integration every element $d \omega$ of the solid angle is to be multiplied by the cosine of the angle it makes with the normal to the area $d S$.

As the same theorem holds for the elementary areas $P_{2}$ and $P_{3}$, etc., it holds for their sum, and hence for a finite surface $S_{2}$ (Fig. I 2). Hence we see generally that the luminous flux due to a surface 
$S_{1}$ passing through $S_{2}$ is equal to the luminous flux due to $S_{2}$ passing through $S_{1}$, the brightness being the same in each case. This is analogous to the theorem that the magnetic flux due to a magnetic shell $S_{1}$, which passes through a second shell $S_{2}$, is equal to that part of the magnetic flux of $S_{2}$ which passes through $S_{1}$, the strength of the shells being supposed the same. Or, again, the number of lines of force due to unit current in an electric circuit $S_{1}$ passing through $S_{2}$ is equal to the number of lines of force due to unit current in $S_{2}$ passing through $S_{1}$. It follows from the above that in any closed surface of uniform brightness the flux passing out from any portion $S_{1}$ is equal to that received from the remainder of the surface $S_{2}$.

\section{HOLLOW SPHERE ${ }^{7}$}

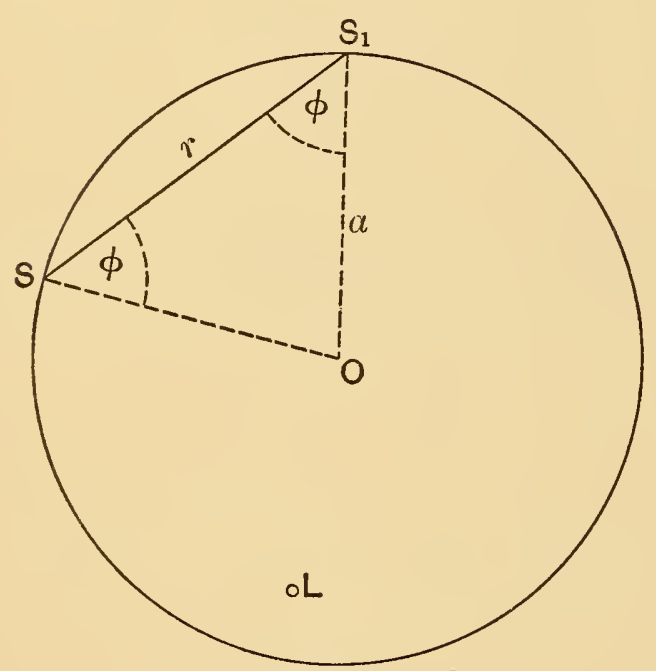

Fig. 13.

Suppose a hollow sphere (Fig. I3) of uniform surface having a coefficient of diffuse reflection $m$,

$$
\text { I }-m=\text { absorption. }
$$

Let $E=$ illumination at $S$.

$$
\begin{aligned}
& E^{\prime}=m E=\text { radiation from } S . \\
& b=\frac{m E}{\pi}=\text { brightness of } S .
\end{aligned}
$$

The flux falling on $S_{1}$ due to $S$ is

$$
\left.\begin{array}{rl}
S_{1} d E_{1} & =\frac{e S S_{1} \cos ^{2} \phi}{r^{2}}=\frac{m E}{\pi} \cdot \frac{S S_{1} \cos ^{2} \varphi}{r^{2}} \\
\text { But } r & =2 a \cos \phi \\
r^{2} & =4 a^{2} \cos ^{2} \phi \\
\frac{\cos ^{2} \phi}{r^{2}} & =\frac{1}{4 a^{2}}
\end{array}\right\} \therefore d E_{1}=\frac{m E}{\pi} \cdot \frac{S}{4 a^{2}}
$$

and this is the same for every element of the sphere. Hence every element illuminates all other elements equally. Therefore the indirect illumination of the sphere must be the same everywhere, no matter how unequal the direct illumination may be. That is, 
a light at $L$ illuminates the sphere unequally, directly. But that part of the total illumination due to diffuse reflection is, notwithstanding, everywhere equal.

A light of mean spherical intensity $I$ sends out $4 \pi I$ lumens.

Of this there is reflected, Ist, $4 \pi m I$ lumens.

$$
\begin{aligned}
& \text { " " " " " " } 2 \mathrm{~d}, 4 \pi m^{2} I \text { lumens. } \\
& \text { " " " " " " } " 3 \mathrm{~d}, 4 \pi \mathrm{m}^{3} I \text { lumens, and so on. }
\end{aligned}
$$

Therefore total amount of flux reflected is

$$
4 \pi I m\left[\mathrm{I}+m+m^{2}+m^{3}+\ldots\right]=4 \pi I \frac{m}{\mathrm{I}-m}=F_{2}
$$

Hence the secondary illumination everywhere equal on the surface of the sphere is

$$
E_{2}=\frac{F_{2}}{4 \pi a^{2}}=\frac{m I}{(\mathrm{I}-m) a^{2}}
$$

Thus the indirect illumination is proportional to $I$, and the lamp of intensity $I$ may be anywhere in the sphere. It is equal to $\frac{m}{\mathrm{I}-m}$ of what the direct illumination would be if the source were placed at the center of the sphere. For example, let a 16 candlepower lamp be placed within a sphere having a radius of I meter and a coefficient of diffuse reflection of 0.8 .

$$
\text { Then } \begin{aligned}
I & =\mathrm{I} 6 \\
a & =\mathrm{I} \text { meter } \\
m & =0.8 \\
E_{2} & =\frac{0.8}{0.2} \frac{\mathrm{I} 6}{\mathrm{I}}=64 \text { meter-candles } \\
E_{1} & =\frac{I}{a^{2}}=\mathrm{I} 6 \text { meter-candles, if lamp is in the center } \\
E & =E_{1}+E_{2}=80
\end{aligned}
$$

Thus the total illumination is five times what it would be if the walls were perfectly black. We can put this in another way: Of the total illumination of 80 meter-candles 20 per cent is absorbed by the walls. Therefore the lamp or source must supply only one-fifth of the total, just enough to make good the constant loss.

Thus the source is analogous to an exciter of electric waves that must supply just enough energy to make good the resistance losses in the circuit. 


\section{LUMINOUS FLUX WITHIN AN INCLOSURE}

If the inner surface of the hollow sphere has a brightness $b$ and a specific radiation $E^{\prime}=\pi b$, a unit disk at the center of the sphere

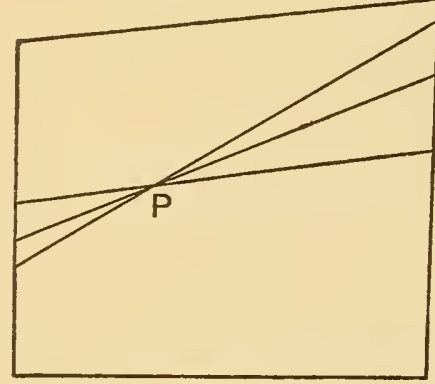

Fig. 14. will receive an illumination $E=\pi b$, page 559. The same will be true wherever the unit disk is placed within the sphere and whatever the orientation of the disk; that is, the flux falling on the disk will be everywhere the same. The flux density within the hollow sphere is therefore everywhere uniform and equal to $\pi b$. The flux from a point source is thought of as in straight lines, and a disk can be placed normal to the direction of the flux. But within the sphere the flux has a uniform value, but no resultant direction.

Within a cube or enclosure of any shape, of which the walls have a uniform brightness $b$ or uniform specific radiation $E^{\prime}$ the same condition obtains as in the sphere-namely, the luminous flux is everywhere the same, and a small area will have the same illumination no matter where it is placed or how it is oriented. This is seen by dividing up the space about any point $P$ (Fig. I4) into elementary solid angles. The illumination due to the surface subtending an angle $\omega$ is independent of the distance from $P$, and hence it will be $\pi b$ for the total angle $2 \pi$ on either side of the surface at $P$, no matter where the surface is placed.

The same is true, therefore, for the space between two infinite planes of brightness $b$. The illumination is $\pi b$ on a small plane at $P_{1}, P_{2}$, or $P_{3}$ (Fig. I 5), anywhere between the two radiating planes $S$ and $T$ no matter how they may be placed. Evidently we can

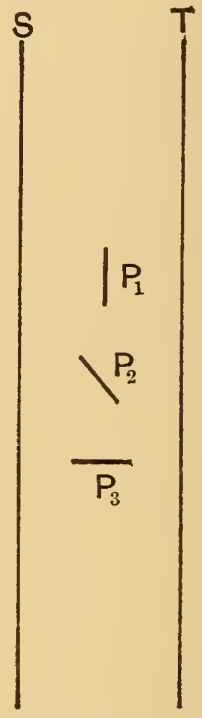

Fig. 15 not think of the flux as normal to the planes, as with the lines of force due to electrostatic charges on the planes $S$ and $T$. The luminous flux normal to $P_{3}$ is the same as normal to $P_{1}$. On the other hand, the electric force normal to $P_{3}$ would be zero.

These theorems have a practical application in the lighting of rooms. 


\section{SUMMARY OF PHOTOMETRIC RELATIONS}

The preceding discussion has shown the necessity for distinguishing several different photometric quantities which are sometimes confused. In order to fix our ideas more clearly, it will be advantageous now to state as concisely as possible the definitions of the several quantities and distinctions between them.

Luminous flux, or light as the term is used in photometry, is the usual physical stimulus which excites vision. It is propagated by means of the vibratory motion in the ether, and the frequency of the vibrations or the combination of frequencies present in any given case determines the color. The total quantity of flux $F$ flowing away from a monochromatic luminous source is proportional to the total radiant energy (per second) and to a stimulus coefficient, the latter being the luminous efficiency $K_{\lambda}$ for the particular frequency or wave length of the given radiation. Thus the equations

$$
\begin{aligned}
& F=K_{\lambda} W \\
& K_{\lambda}=\frac{F_{\lambda}}{W_{\lambda}}
\end{aligned}
$$

express the luminous flux as the power $W$ multiplied by the luminous efficiency $K_{\lambda}$, and if flux is expressed in lumens and the power in watts, the luminous efficiency is the number of lumens per watt of radiation of the wave length $\lambda$. For white or chromatic light $K$ will have a value depending on the distribution of the energy in the spectrum. It is a maximum in the yellow-green region and falls off rapidly in either direction, reaching zero at the limits of the visible spectrum. The luminous efficiency of most light sources is greatly reduced by the amount of radiation outside the visible spectrum, chiefly of longer wave length than that of visible radiation, and the total efficiency of such a source

$$
K=\frac{F}{W}
$$

is the quotient of the total luminous flux divided by the total radiant power.

For the purposes of definition and of expressing the mathematical relations involved in photometry, it is permissible to confine ourselves to monochromatic light and to consider $K$ a constant, 
although it does in fact vary somewhat with the magnitude of the flux density. We also assume that all surfaces are perfectly diffusing and obey the cosine law and that there is no absorption in the atmosphere.

The intensity of a point source or uniform luminous sphere is measured by the luminous flux flowing through a unit solid angle whose apex is the given point or center of the given sphere. Thus from a source of intensity $I$ light is flowing away at a rate of $I$ lumens per unit solid angle or a total of $4 \pi I$ lumens for the point source or uniform sphere. If the source is not uniform and light is flowing away at unequal rates in different directions, the intensity $I$ in any direction is equal to the flux $d F$ in an elementary solid angle $d \omega$ taken in the given direction. Thus

$$
I=\frac{d F}{d \omega}
$$

is a general expression applying to all point sources whether radiating equally or unequally in different directions. If the unsymmetrical source is extended-as, for example, an incandescent lamp or a diffusing globe-the same holds true if the distance at which the measurements are made are sufficiently great so that the distribution of light is practically the same as from an unsymmetrical point source. For less distances than this the intensity is not a constant in a given direction, but varies with $r$. In this case the equivalent intensity at any point is equal to that of a point source which gives the same flux density, or lumens per sq $\mathrm{cm}$, at the point that the given source does. The mean spherical intensity $I_{s}$ is the average value of the intensity and is equal to the total flux $F$ divided by $4 \pi$.

The total flux from a given extended source is therefore a constant independent of distance, as is also the mean spherical intensity $I_{s}$. The intensity $I$ in a particular direction, however, in the case of extended sources other than spheres varies with the distance, but at relatively great distances the variation is inappreciable. Thus the luminous flux is the fundamental quantity. But while we define $I$ as the flux per unit solid angle, or rate of flux with respect to solid angle, we determine $I$ by comparison with a concrete standard. Thus photometric standards of intensity are standards of light flux, their values being expressed in candles. 
If $f$ is the spherical reduction factor with respect to any particular direction, and $I$ is the intensity of a source in that direction,

$$
I_{s}=f I
$$

For a unit disk-that is, a small circular disk of uniform brightness - the total flux is $\pi$ times the normal intensity $I_{n}$, whereas the mean spherical reduction factor with respect to the normal is onefourth. Hence, the total flux is

$$
\begin{aligned}
F & =\pi I_{1} \\
& =4 \pi I_{s}, \text { as for a sphere. }
\end{aligned}
$$

In general, for any light source, $F_{s}=4 \pi I_{s}=4 \pi f I$, but for extended sources other than spheres, the value of $f$ as well as $I$ varies with the distance from the source for points relatively near the source.

The specific flux or flux density is the luminous flux per unit of area, or lumens per square centimeter. When the flux falls upon a material surface, we call the specific flux the illumination, $E$. When we speak of the flux coming from a surface, whether it be a self-luminous source at high temperature or a reflecting or radiating surface at low temperature, we call the specific flux the specific radiation, or simply the radiation, $E^{\prime}$.

Thus the illumination $E$ is

$$
E=\frac{F_{i}}{S}=\frac{d F_{i}}{d S}=\frac{I}{r^{2}}
$$

The radiation $E^{\prime}$ is

$$
E^{\prime}=\frac{F_{e}}{S}=\frac{d F_{e}}{d S}
$$

$F_{i}$ is the incident flux, $F_{e}$ is the emitted or radiated flux. If $m$ is the coefficient of diffuse reflection or transmission, $(I-m)$ being the absorption,

$$
\begin{aligned}
& F_{e}=m F_{i} \\
& E^{\prime}=m E
\end{aligned}
$$

where the radiation consists in the diffuse reflection or transmission of a portion of the incident flux or illumination.

The radiation or illumination when large may be expressed in lumens per sq cm; when small in milli-lumens per sq $\mathrm{cm}$. The milli-lumen per sq $\mathrm{cm}$ is nearly equivalent to the foot candle. 
I lumen per sq $\mathrm{cm} \quad=$ Io ooo lumens per sq meter.

$=\mathrm{Io} 000$ meter-candles.

$\therefore$ I milli-lumen per sq $\mathrm{cm}=$ Io meter-candles $=$ Io lux.

$$
=\frac{\mathrm{I}}{\mathrm{I} .0765} \text { foot-candles. }
$$

The brightness $b$ of a source is the intensity in candles per sq $\mathrm{cm}$ of area, taken normally. Thus

$$
b=\frac{I}{S}=\frac{d I}{d S}=\frac{d Q}{d S}
$$

Brightness, or specific quantity, refers to the quantity of light per unit of area of a source, and is measured in candles per sq $\mathrm{cm}$. Brightness can refer equally to luminous sources of relatively high specific intensity or to reflecting and radiating sources of low intensities. The latter may be conveniently expressed in milli-lumens per sq cm. Thus we may say a flame has a specific radiation of ro lumens per sq $\mathrm{cm}$ or a brightness of 0.8 candles per sq cm; and a wall has a specific radiation of to milli-lumens per sq cm, or a brightness of 0.8 milli-candles per sq $\mathrm{cm}$ or of 8 candles per sq meter.

The quantity $Q$ is proportional to the total amount of light emitted by the source, and is equal to the surface integral of the brightness $b$. Thus

$$
Q=\int b d S
$$

The quantity for a small luminous circular disk of radius $a$ and uniform brightness $b$ is

$$
Q=\pi a^{2} b=1_{n}
$$

That is, the quantity is equal to the maximum intensity. In this case the whole surface is equally effective in producing the illumination on the test screen by which the intensity $I_{n}$ is measured. But for an extended disk, the quantity and the normal intensity, as we have seen above, are not the same. Thus, the quantity is $b$ times the surface, or 


$$
\begin{aligned}
Q & =\pi a^{2} b \\
E_{n} & =\frac{Q}{a^{2}+r^{2}}=\frac{I_{n}}{r^{2}} \\
\therefore \frac{I_{n}}{Q_{1}} & =\frac{r^{2}}{a^{2}+r^{2}}=\frac{r^{2}}{d^{2}}=\cos ^{2} \theta
\end{aligned}
$$

That is, the normal equivalent intensity $I_{n}$ of the disk (Fig. I6) with respect to the point $P$ on the axis of the disk is $Q$ times $\cos ^{2} \theta$. When the distance is equal to the radius of the disk, the quantity $Q$ is twice the normal intensity $I_{n}$.

The total luminous flux is $\pi b S$ or $\pi$ times the quantity, and the

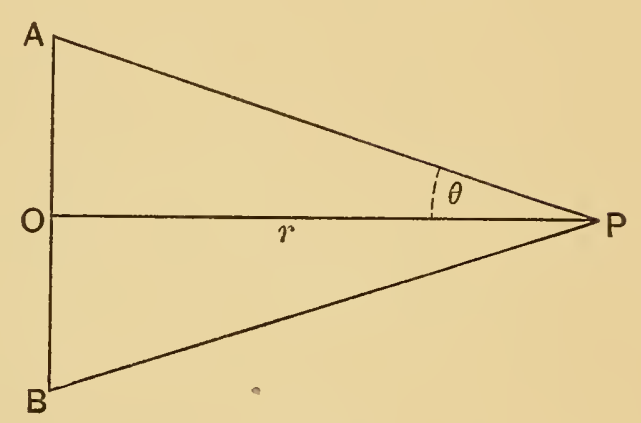

Fig. 16. mean hemispherical intensity is $\frac{Q}{2}$ or half the quantity.

In the case of a sphere of uniform brightness $b$ the quantity is $\int b d S=4 \pi a^{2} b$. The intensity $I=\pi a^{2} b$. Hence the intensity is onefourth the quantity. In other words, the total radiation from the sphere is four times as great as from a unit disk of the same normal intensity. The relations between quantity and intensity for a few simple cases are as follows:

For a unit disk $I_{n}=Q$.

For an extended circular disk $I_{n}=Q \cos ^{2} \theta=Q \frac{r^{2}}{d^{2}}$.

For a sphere $I=\frac{\mathrm{I}}{4} Q$.

For a unit cylinder $I_{1}=\frac{\mathrm{I}}{\pi} Q$.

The total luminous flux delivered in a given time-that is, the time integral of the luminous flux-may be expressed in lumen-seconds or lumen-hours, according to circumstances. Thus, putting $L$ for the total lighting in the time $T$

$$
\begin{aligned}
L & =F T \\
& =\int F d T, \text { if } F \text { is variable }
\end{aligned}
$$

where $F$ is in lumens and the time is expressed in the most convenient unit. The flash of a firefly may be expressed in lumen- 
seconds; the total lighting per gram of an illuminant, or the total lighting given during the life of an incandescent lamp, is better expressed in lumen-hours.

Since flux of light may also be expressed in spherical candles ( $\frac{\mathrm{I}}{4 \pi}$ times the lumens), we may also express the time integral or total lighting in terms of spherical candles and hours. Thus $L=I_{s} T=\int I d T$, if the spherical candlepower is a variable with respect to $T$, the value of $L$ being here given in candle-hours.

The photometric quantities employed in the preceding discussion are shown in Table I, together with the units in which they are expressed and the equations of definition.

TABLE I

\begin{tabular}{|c|c|c|c|}
\hline Photometric Magnitude & Symbol & Unit & Equation of Definition \\
\hline I. Intensity of light & $I$ & Candle & $I=\frac{F}{\omega}$ \\
\hline 2. Luminous flux & $F$ & Lumen & $F=I \omega=\frac{I S}{r^{2}}=E S=\pi Q$ \\
\hline 3. Illumination & $E$ & $\left\{\frac{\text { Lumens }}{\mathrm{cm}^{2}}\right.$ or $\frac{\text { milli-lumens }}{\mathrm{cm}^{2}}$ & $E=\frac{F_{i}}{S}=\frac{I}{r^{2}}$ \\
\hline 4. Radiation & $E^{\prime}$ & $\mathrm{Lux}=$ meter-candle & $E^{\prime}=\frac{F_{e}}{S}=\pi b=m E$ \\
\hline 5. Brightness & $b$ & $\frac{\text { Candles }}{\mathrm{cm}^{2}}$ & $b=\frac{1}{S \cos e}$ \\
\hline 6. Quantity & $Q$ & Candles & $Q=b S$ \\
\hline 7. Lighting & $L$ & Lumen-hours & $L=F T$ \\
\hline
\end{tabular}

$I, b, Q$ are expressed in candles. $F, E, E^{\prime}$ are expressed in lumens.

$L$ is in lumens or spherical candles. $E^{\prime}=\pi . b=m E$. $F=\pi Q$.
$F_{i}=$ incident flux.

$F_{e}=$ emergent flux.

$m=$ coefficient of diffuse reflection or transmission.

$(I-m)=$ coefficient of absorption.

The symbol $F$ has been employed for the flux (as originally proposed by Hospitalier) instead of $\Phi$, for the following reasons:

I. $\Phi$ is the only Greek letter in the series, and it is more consistent to use a Latin letter; $F$ is the initial letter of the word flux.

2. The letter $\Phi$ is more or less unfamiliar to many illuminating engineers and also to many printing offices, and it is often confused with the small letter $\phi$, which is used for an angle. 
The symbol $E^{\prime}$ is used for radiation instead of $R$ (as proposed by Hospitalier), because it is so closely related to the illumination, and because the letter $R$ is employed for the distance from the source. Blondel and others proposed to employ the same letter, $E$, for illumination and radiation, but that gives rise to confusion. On the other hand, $E^{\prime}$ gives sufficient distinction and at the same time recalls their close connection. The letter $b$ is used for brightness instead of $i$ for specific intensity because $i$ is used for the angle of emission, and specific intensity is a less desirable name. The quantity is a specific intensity strictly only for small plane areas, not for small spheres or large sources of any form. Quantity of light, $Q$, is here used as the surface integral of $b$ instead of the time integral of $F$. It is analogous to quantity of electricity in electrostatics and is more properly employed in the sense here used than with the other meaning. The term lighting for flux times time is use in harmony with the usage in France and Germany.

\section{PROBLEMS FOR ILLUSTRATIONS}

Problem I.-A lamp of 200 candlepower (supposed uniform in all directions) is placed in the center of a spherical diffusing globe of $40 \mathrm{~cm}$ diameter, the absorption of which is 30 per cent. Required, the intensity of the globe, its brightness, its specific radiation, the illumination on its inner surface, and the illumination it produces at a distance of 3 meters from the center of the globe.

The illumination on its inner surface is $E=\frac{I}{a^{2}}=\frac{200}{400}=0.5$ lumens per sq $\mathrm{cm}$ (formula I). The radiation $E^{\prime}$ is $m E$, where $m$ is one minus the absorption; it is here 0.7 . Therefore the radiation is 0.35 lumens per sq $\mathrm{cm}$. The brightness $b$ is $\frac{E^{\prime}}{\pi}$ or $0.1 \mathrm{I} 2$ candles per sq $\mathrm{cm}$. The intensity $I$ of the globe is $200 \times 0.7=140$ candles. The illumination $E$ at a distance of 3 meters is

$$
\begin{aligned}
E=\frac{\mathrm{I} 4 \mathrm{O}}{300^{2}} & =.00 \mathrm{I} 56 \text { lumens per sq cm } \\
& =\mathrm{I} .56 \text { milli-lumens per sq cm } \\
& =\mathrm{I} .45 \text { foot-candles } \\
\text { or } \mathrm{E}=\frac{\mathrm{I} 4 \mathrm{O}}{3^{2}} & =\mathrm{I} 5.6 \text { meter-candles } \\
& =\mathrm{I} 5.6 \mathrm{lux} .
\end{aligned}
$$


Problem 2.-A circular area $S, 2$ meters in diameter, on the side of a wall is uniformly illuminated, $E$ being 4 meter-candles. A photometer placed I meter from the wall, perpendicular to the center of the illuminated area, measures the equivalent intensity $I_{0}$ of the area $S$, and finds it to be I candle. What is the absorption coefficient of the wall?

The illumination $E$ being 4 meter-candles, and the area $S$ being $\pi$ square meters, the flux $F$ falling on the area $S$ is $4 \pi$ lumens. The measured intensity $I$ at a distance $r=\mathrm{I}$ meter is I candle. Therefore, the quantity of light on the disk is

$$
Q=I_{0} \frac{d^{2}}{r^{2}}=\mathrm{I} \times \frac{2}{\mathrm{I}}=2 \text { candles }
$$

The total flux from the disk is $\pi$ times the quantity $Q$. Therefore, the total flux coming from the area $S$ is $2 \pi$ lumens, whereas the flux falling upon it is $4 \pi$ lumens. Therefore, the coefficient of absorption is $\frac{I}{2}$ or 5 o per cent.

Problem 3.- Suppose a room of 900 square meters total wall surface is to be so lighted that the walls shall have an average illumination of ro lumens per square meter, the coefficient of absorption of the walls being 40 per cent on the average. How many lamps of I 5 mean horizontal candlepower will be required?

Part of the illumination will be due to light reflected from the walls. The lamps must supply that which is absorbed. The flux to be supplied is therefore $F=0.40 \times 900 \times 10=3600$ lumens. If each lamp has a spherical reduction factor of 80 per cent, it will supply $4 \pi \times 0.80 \times 15=$ I 50 lumens, approximately. Hence, 24 lamps will be required.

(Examples I and 3 are borrowed from one of Blondel's papers.) 


\section{COLLECTION OF FORMULAS}

I. $E=\frac{I}{r^{2}}$ for point source, unit sphere or sphere of any size.

$I=\pi a^{2} b$, where $a=$ radius of sphere and $b=$ brightness of surface.

2. $E=\frac{\pi a^{2} b}{r^{2}}$ for sphere of radius $a$.

$=\pi b$ when $r=a$; that is, at the surface of the sphere, same as for an infinite plane.

3. $E=\frac{\pi a^{2} b}{a^{2}+r^{2}}=\frac{Q}{d^{2}}$ for disk of radius $a$, at distance $r$ on axis.

$d=$ distance of point on axis to edge of disk.

4. $E=\frac{\pi a b}{r}$ for infinite cylinder, $b=$ brightness, $a=$ radius.

$=\frac{Q_{1}}{2 r}$, where $Q_{1}=$ quantity of light per unit of length

$=\pi b$ at surface. $I_{1}=\frac{2 a b}{r^{2}}=$ intensity per unit of length

5. $E=\pi b$ for infinite plane, at all distances.

6. $E=\frac{b d S \cos e}{r^{2}}=b d \omega$ for any small surface $d S$ subtending a small angle $d \omega$, at any distance.

7. $E=\frac{2 b}{r} \cos e$, for infinitely long, very narrow strip of $b$ units of light per unit of length

$$
=2 \frac{Q_{1}}{r}
$$

8. $Q=\int b d S$ over sphere, cylinder, disk or other surface where $b=$ normal intensity $=$ quantity of light per unit of area. $48848^{\circ}-10-$ - 10 
9. $F_{s}=\pi Q$, for sphere or other extended source

I0. $E=\frac{Q}{d^{2}}=\frac{I_{0}}{r^{2}} \therefore \frac{I_{0}}{Q}=\frac{r^{2}}{d^{2}}=\cos ^{2} \theta$, for a disk.

$I_{0}=$ equivalent point source, $Q=$ quantity of light over disk $I=Q / 4$ for a sphere.

WASHINGTON, May io, I9io.

The letter $b$ is used for brightness instead of $e$ as was done in an earlier paper in the Transactions of the Illuminating Engineering Society in accordance with the preference of the Committee on Nomenclature, following the suggestion of Prof. Blondel. 